\title{
Regulation of Bcl-2 and the NF-kB Signaling Pathway by Succinyl Rotundic Acid in Livers of Rats with Alcoholic Hepatitis
}

\author{
Yufang $\mathrm{He}^{1}$, Fang Xia ${ }^{1}$, Minlun $\mathrm{Nan}^{2}$, Lijing $\mathrm{Li}^{1}, \mathrm{Xu} \mathrm{Wang}^{3}$ and Yajie Zhang ${ }^{{ }^{*}}$ \\ ${ }^{1}$ Changchun University of Chinese Medicine, Changchun 130117, China \\ ${ }^{2}$ Jilin Academy of Chinese Medicine Sciences, Changchun 130012, China \\ ${ }^{3}$ Changchun infectious disease hospital, Changchun 130118, China \\ *For correspondence: zyjcczyy@163.com \\ Received 23 September 2020 ; Accepted 09 November 2020 ; Published 25 January 2021
}

\begin{abstract}
In this study, the protective effects of succinyl rotundic acids on alcoholic hepatitis in irradiated rats as well as the effects of Bcl-2-Bax-caspase-3 and the NF-kB signal pathways were studied. SD rats were divided into four groups randomly: normal; model; and succinyl rotundic acid low-, middle-, and high-dose groups. Distilled water, $60 \%$ ethanol and 60\% ethanol +SRA, respectively, were given for 30 days. ELISA was used to measure serum levels of LDH, AST, ALT, NOS, NO, MDA, GSH and TG. Western blotting was used to measure protein levels of Bcl-2, Bax, caspase-3, NF-kB p 65, IKBA, HO-1, Nrf2 and CYP2E1. Compared with the model group, LDH, AST, ALT, NOS, NO, MDA and TG levels were lower in serum of low-, middle-, and high-dose groups $(P<0.01, P<0.05$ and $P<0.05$ in all); GSH content was greater in serum of low-, middleand high-dose groups $(P<0.05)$. Levels of Bcl-2, HO-1, and Nrf2 were greater $(P<0.01$ in all $)$; those of Bax, caspase-3, NFkB p65, IKBA, and CYP2E1 were lower $(P<0.01$ and $P<0.001$ in all). These findings suggest that succinyl rotundic acid reduces inflammatory reactions by reducing levels of NOS and NO, regulating levels of Bcl-2, Bax, caspase-3, NF-kB, and anti-oxidative stress pathways, and has an antagonistic effect on alcoholic liver injury. The agent has potential to treat clinical alcoholic liver disease. (C) 2021 Friends Science Publishers
\end{abstract}

Keywords: Hepatitis rats; Protective effect; Serum levels; Western blotting

\section{Introduction}

Alcoholic liver disease (ALD) is caused by long-term heavy drinking (Li et al. 2011). It usually manifests as fatty liver in the initial stage and develops into alcoholic hepatitis, cirrhosis and hepatic fibrosis. There is a lack of large-scale epidemiological survey data on alcoholic liver disease in China (Wei et al. 2015; Ciardullo et al. 2020).

The first-line therapy for severe alcoholic hepatitis is corticosteroids; however, the evidence for its effectiveness in reducing mortality remains unclear. Pentoxifylline is an alternative therapy (Maryconi and Mitchell 2014). Succinyl rotundic acid has pharmacological effects including antioxidation, anti-inflammation, scavenging oxygen free radicals, and others. It treats liver diseases such as acute liver injury, nonalcoholic fatty liver, liver fibrosis, liver cancer, and others. In view of these problems, our research group designed succinyl rotundic acid (Li et al. 2017) to treat alcoholic liver disease.

This study established a chronic ethanol-induced liver injury model in rats, studied its hepatoprotective effects, explored the relevant mechanisms, and measured liver function and liver tissue oxidation injury indexes to evaluate the therapeutic effects of succinyl rotundic acid (Hsu et al. 2015) on alcoholic liver disease.

\section{Materials and Methods}

\section{Materials sample}

Succinyl rotundic acid (SRA) was obtained by chemical modification of RA. RA was heated in the presence of pyridine and reacted with succinic anhydride. Cooling to room temperature, after the completion of an action in ice water, dilute hydrochloric acid was added to adjust the $\mathrm{pH}$ to 4-. After filtration, the filter residue was washed with water until it was colorless. The final SRA product was obtained by recrystallizing. The purity of SRA was $95.70 \%$ by HPLC.

Sprague Dawley (SD) rats, male, weights 180-220 g were used. After adaptive feeding, 60 SD rats were divided into normal, model, succinyl rotundic acid low-dose (10 $\mu \mathrm{mol} / \mathrm{L})$, middle-dose $(20 \mu \mathrm{mol} / \mathrm{L})$, and high-dose (40 $\mu \mathrm{mol} / \mathrm{L})$ groups according to body weight. In the normal 
group, the rats were given equal volume distilled water. Except for the normal group, rats in each group were given $10 \mathrm{~mL} / \mathrm{kg}$ of $60 \%$ ethanol solution orally every day. After 6 $\mathrm{h}$, the drug group was given the corresponding drug orally according to the designed dose $\left(2.0 \mathrm{~mL} / \mathrm{kg}^{-} \mathrm{d}\right)$ for 30 days. The model group was not given drugs. After the final administration, the rats were fasted for $16 \mathrm{~h}$ without withholding drinking water, weighed, and blood and liver tissues were taken for each index test.

\section{LDH, AST, ALT, NOS and NO measurement}

Serum was separated and levels of lactate dehydrogenase (LDH), aspartate transaminase (AST) and alanine aminotransferase (ALT) were determined using an automatic blood biochemical analyzer. The activities of nitric oxide synthase (NOS) and nitric oxide (NO) were determined by a chemical chromogenic method according to the kit instructions.

\section{MDA, GSH and TG measurement}

Liver tissue was homogenized according to kit instructions. The levels of malondialdehyde (MDA), glutathione (GSH) and triglyceride (TG) in liver tissue were measured according to the kit instructions.

\section{Western blotting}

Liver tissue homogenates was prepared using RIPA to obtain protein samples. A BCA protein concentration determination kit was used to measure protein concentrations. After SDS-PAGE electrophoresis, proteins were transferred to polyvinylidene fluoride (PVDF) membranes and washed in TBST. Membranes were incubated with antibodies to Bcl-2 (1:700), Bax (1:2000), caspase-3 (1:700), NF-kB p 65 (1:2000), IKBA (1:2000), HO-1 (1:2 000), Nrf2 (1:2000), CYP2E1 (1:2000), and $\beta$ actin $(1: 2000)$ overnight at $2-8^{\circ} \mathrm{C}$; then added goat antirabbit secondary antibody (1:2000). Detection, exposure, development and fixation involved ultra-sensitive enhanced chemiluminescence (ECL) reagents. Gel-Pro-Analyzer software (Media Cybernetics Inc.) was used to measure the optical density.

\section{Statistical analysis}

The data was expressed as $\mathrm{M} \pm \mathrm{S}$. Use Bonferroni post-hoc test for multiple comparisons and one-way ANOVA was used for inter-group comparison. Use GraphPad Prism 5.0 software to process data and numbers.

\section{Results}

\section{Body weight}

The initial body mass in each group of rats showed no significant differences before intragastric administration. After 30 days of continuous gavage, the body weights in the normal group were higher than those of other groups, however there without significance (Table 1 and Fig. 1A).

\section{Content of LDH, AST, ALT, NOS and NO in liver tissue}

LDH, AST, NOS and NO levels in the model group were significantly greater differences $(P<0.01, P<0.05, P<$ 0.001 , and $P<0.001$, respectively) than those of the the normal group. Serum LDH levels in the low-, middle-, and high-dose groups were significantly lower than those of the model group $(P<0.01, P<0.001$, and $P<0.001$, respectively, Fig. 1B); AST content in serum was significantly lower in the three groups $(P<0.05, P<0.01$, and $P<0.01$, respectively, Fig. 1C). Serum ALT levels in the low-, middle-, and high-dose groups were significantly lower $(P<0.05, P<0.05$ and $P<0.01$, respectively, Fig. 2A). Serum levels of NOS and NO were significantly lower in the middle- and high-dose groups $(P<0.05$ and $P<0.01$, respectively, Fig. 2B-C and Table 2).

\section{Changes of MDA, GSH and TG in liver tissue}

Levels of MDA and $\mathrm{TG}$ in the model group were significantly higher, while levels of GSH were significantly lower $(P<0.001, P<0.05$, and $P<0.01$, respectively) than those of the normal group. MDA levels in liver tissue were significantly lower in the middle-, and high-dose groups $(P$ $<0.05$ and $P<0.01$, respectively Fig. 3A), GSH levels in liver tissue were significantly higher in the high-dose group $(P<0.05$, Fig. 3B), TG levels in liver tissue were significantly lower in the high-dose group $(P<0.05$, Fig. $3 \mathrm{C}$ and Table 3) than in the model group.

\section{Western blotting}

Liver levels of Bcl-2 in the model group were significantly lower $(P<0.001)$, and levels of Bax and caspase-3 were significantly greater $(P<0.001$ in all $)$ than in the normal group. Levels of $\mathrm{Bcl}-2$ in liver in the high-dose group were significantly greater $(P<0.01)$, and levels of Bax and caspase-3 in the high- and middle-dose groups were significantly lower $(P<0.01$ and $P<0.05$, respectively) (Fig. 4A-B) than in the model group.

Western blotting revealed that levels of CYP2E1 in liver tissue in the model group were significantly greater than those of the normal group $(P<0.001)$. The high-, middle-, and low-dose groups showed significantly lower levels of CYP2E1 $(P<0.001, P<0.01$, and $P<0.05$ respectively) (Fig. 4C-D) than those of the model group. These findings suggest that succinyl rotundic acid inhibits hepatic levels of CYP2E1 in rats with alcoholic hepatitis, thereby promoting the rapid decomposition of ethanol and reducing ethanol-induced damage to the liver.

Hepatic levels of Nrf2 in the model group were significantly lower than those of the normal group $(P<$ 
He et al. / Intl J Agric Biol, Vol 25, No 3, 2021

Table 1: Body weights and body weight increase of rats in various groups $(x \pm s)$

\begin{tabular}{lllll}
\hline Group & $\mathrm{n}$ & \multicolumn{2}{c}{ Body weight } & Weight increase \\
\cline { 3 - 4 } & & Before experiment & After experiment & $64.75 \pm 11.25$ \\
Blank control & 12 & $193.56 \pm 4.78$ & $258.31 \pm 5.96$ & $29.91 \pm 10.32$ \\
Model control & 10 & $195.24 \pm 5.26$ & $225.15 \pm 7.82$ & $49.66 \pm 9.68$ \\
Low dose & 10 & $196.23 \pm 4.32$ & $245.89 \pm 6.15$ & $48.78 \pm 10.48$ \\
Middle dose & 11 & $195.34 \pm 3.18$ & $244.12 \pm 5.93$ & $49.25 \pm 11.24$ \\
High dose & 11 & $196.54 \pm 5.69$ & $245.79 \pm 6.48$ & \\
\hline
\end{tabular}

Table 2: Levels of serum LDH, AST, ALT, NOS, NO in alcohol liver injury of rats $(x \pm s)$

\begin{tabular}{|c|c|c|c|c|c|}
\hline Groups & LDH (U/L) & AST (U/L) & ALT (U/L) & NOS (U/mL) & $\mathrm{NO}(\mu \mathrm{mol} / \mathrm{L})$ \\
\hline Blank control & $1015.26 \pm 105.48$ & $105.46 \pm 17.28$ & $32.78 \pm 5.29$ & $19.56 \pm 3.48$ & $20.47 \pm 3.47$ \\
\hline Model control & $1534.15 \pm 114.25 * *$ & $157.68 \pm 24.31 *$ & $45.37 \pm 4.89$ & $34.75 \pm 2.09 * * *$ & $35.28 \pm 2.08 * * *$ \\
\hline Low dose & $1089.24 \pm 123.14^{\& \&}$ & $101.57 \pm 12.36^{\&}$ & $31.36 \pm 5.19^{\&}$ & $28.47 \pm 2.58^{\#}$ & $30.09 \pm 1.48^{\# \#}$ \\
\hline Middle dose & $947.26 \pm 108.97^{\& \& \&}$ & $93.27 \pm 15.68^{\& \&}$ & $29.48 \pm 3.48^{\&}$ & $25.67 \pm 2.08^{\&}$ & $28.32 \pm 2.05^{\# \&}$ \\
\hline High dose & $908.46 \pm 98.56^{\& \& \&}$ & $87.68 \pm 17.29^{\& \&}$ & $26.18 \pm 5.17^{\& \&}$ & $22.34 \pm 2.81^{\& \&}$ & $25.79 \pm 2.36^{\& \&}$ \\
\hline
\end{tabular}

Note: *Blank control $v s$. Model control; ${ }^{\#}$ Blank control $v s$. dose groups; ${ }^{\&}$ Model control $v s$. dose groups

Table 3: Levels of hepatic MDA, GSH, TG in alcohol liver injury of rats $(x \pm s)$

\begin{tabular}{llll}
\hline Groups & MDA $(\mathrm{nmol} / \mathrm{mg})$ & GSH $(\mathrm{mmol} / \mathrm{mg})$ & TG $(\mathrm{mmol} / \mathrm{g})$ \\
\hline Blank control & $0.62 \pm 0.11$ & $21.15 \pm 2.45$ & $0.31 \pm 0.02$ \\
Model control & $1.42 \pm 0.15^{* * *}$ & $11.34 \pm 1.78^{* *}$ & $0.48 \pm 0.08^{*}$ \\
Low dose & $1.24 \pm 0.23^{\text {\#\# }}$ & $13.52 \pm 1.63^{\#}$ & $0.44 \pm 0.05$ \\
Middle dose & $1.01 \pm 0.10^{\&}$ & $15.69 \pm 2.78$ & $0.38 \pm 0.06$ \\
High dose & $0.85 \pm 0.09^{\& \&}$ & $18.45 \pm 2.05^{\&}$ & $0.33 \pm 0.04^{\&}$ \\
\hline
\end{tabular}

*Blank control vs. Model control; ${ }^{\# B}$ Bank control vs. dose groups; ${ }^{\&}$ Model control vs. dose groups
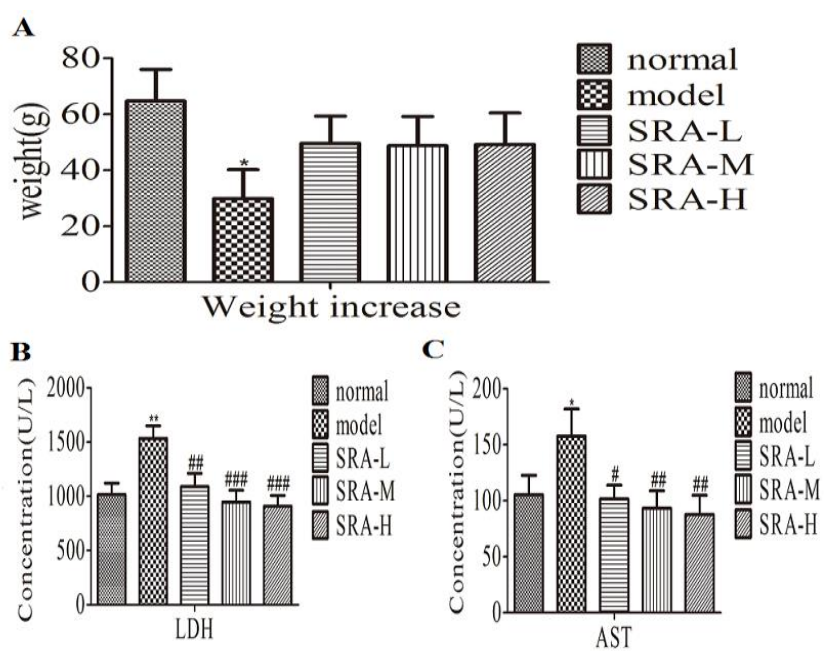

Fig. 1: Body weight changes of SRA on SD rats and effects of SRA on LDH and AST levels in serum. Values are expressed as means \pm SEM. Compared with normal group: ${ }^{*} P<0.05,{ }^{* *} P<$ 0.01 ; compared with model group: ${ }^{\#} P<0.05,{ }^{\# \#} P<0.01,{ }^{\# \#} P<$ 0.001

0.001). The high-dose group showed significantly higher Nrf2 levels $(P<0.01)$ (Fig 4C-D) than those of the model group. These findings suggest that succinyl rotundic acid increases levels of Nrf2 in liver tissue of rats with alcoholic liver injury, increasing the amount of nuclear transfer, further activating the expression of downstream antioxidant

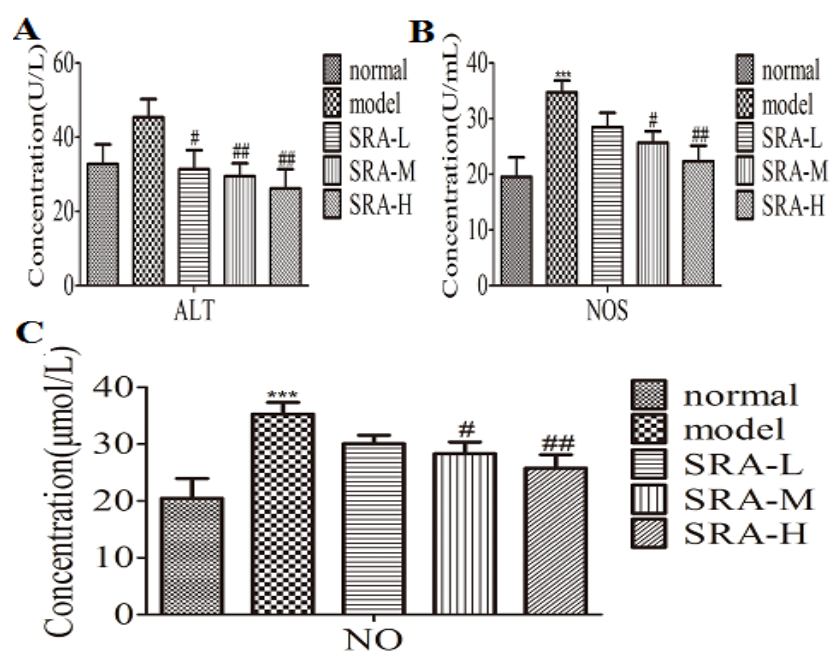

Fig. 2: Effects of SRA on ALT, NOS and NO levels in serum. Values are expressed as means \pm SEM. Compared with normal proteins, and enhancing the oxidation defense system so as to protect the liver.

Levels of HO-1 in liver tissue in the model group were significantly lower $(P<0.001)$ than those of the normal group. The high- and middle-dose groups showed significantly greater levels of HO-1 protein $(P<0.01$ and $P$ $<0.05$, respectively) (Fig. 4C-D) than those of the model group. These findings suggest that succinyl rotundic acid enhances anti-oxidation and anti-apoptosis effects, improving cell survival in liver tissue, and enhancing the 


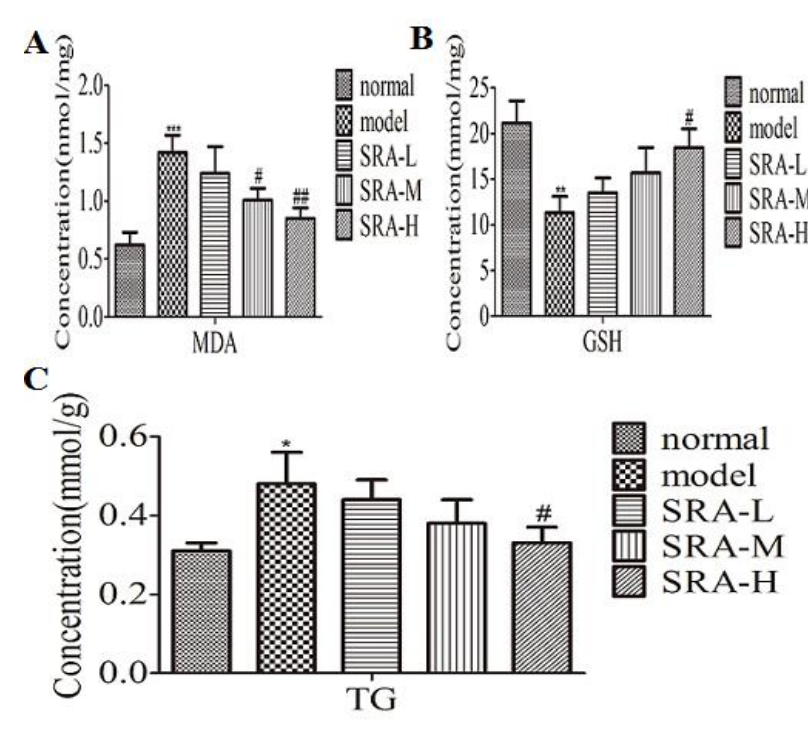

Fig. 3: Effects of SRA on MDA, GSH and TG levels in serum. Values are expressed as means \pm SEM. Compared with normal group: ${ }^{*} P<0.05,{ }^{* *} P<0.01,{ }^{* * *} P<0.001$; compared with model group: ${ }^{\#} P<0.05,{ }^{\#} P<0.01$
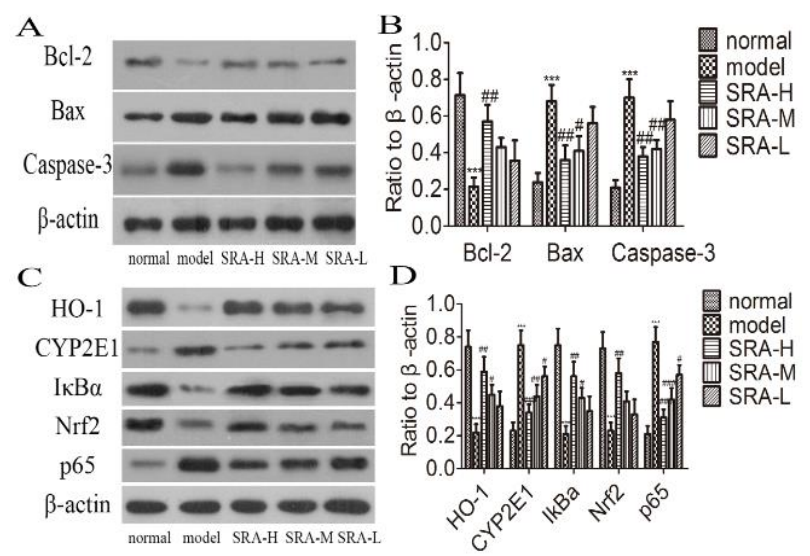

Fig. 4: The effect of SRA on NF- $\kappa \mathrm{B}$ and Bcl-2 signaling pathway in rat. Values are expressed as means \pm SEM. Compared with normal group: ${ }^{* * *} P<0.001$; compared with model group: ${ }^{\#} P<$ $0.05,{ }^{\# \#} P<0.01,{ }^{\# \#+~} P<0.001$

oxidation defense system by up-regulating the expression of antioxidant protein HO-1 in livers of rats with alcoholic hepatitis.

Levels of IKBA in the model group were significantly lower $(P<0.001)$ than those of the normal group. The highand middle-dose group showed significantly greater levels of IKBA than those of the normal group $(P<0.01$ and $P<0.05$, respectively) (Fig. 4C-D). These findings suggest that succinyl rotundic acid inhibits the degradation of IKBA protein in the livers of rats with alcoholic liver injury and reduces the release of NF-KB, thereby inhibiting inflammation.

Levels NF-kB p 65 protein were significantly lower than those of the normal group $(P<0.001)$. Compared with the model group, the high-, middle-, and low-dose groups showed significantly lower levels of NF-kB p $65(P<0.001$, $P<0.001$, and $P<0.05$, respectively) (Fig. 4C-D). These findings suggest that succinyl rotundic acid inhibits levels of NF-kB p 65 protein in liver tissue of rats with alcoholic liver injury, reducing the release of inflammatory factors, and thereby inhibiting inflammation.

\section{Discussion}

In this study, rats in the model group showed significant liver injury after long-term administration of ethanol. Levels of serum LDH, AST, and NOS significantly increased (Baghdasaryan et al. 2019; Sehgal et al. 2020; Wu et al. 2020; Zou et al. 2020), suggesting abnormal liver function and liver cell injury. MDA levels in liver tissue increased significantly and those of GSH decreased, suggesting lipid peroxidation damage in liver tissue. The increase of TG levels in liver tissue suggests fatty degeneration of liver tissue. Succinyl rotundic acid reduced serum levels of LDH, AST, NOS, and NO (Zhang et al. 2002; Tang et al. 2009; He et al. 2021), increasing GSH activity, and reducing levels of MDA and TG (Jiao et al. 2020; Noto et al. 2020), suggesting that it ameliorates alcoholic liver injury and reduces lipid peroxidation damage and steatosis. Levels of Bcl-2 in liver decreased while levels of Bax and caspase- 3 increased (Raisova et al. 2001; Klemm et al. 2008; Mao et al. 2008). Succinyl rotundic acid inhibited expression of CYP2E1 protein in liver of rats with alcoholic hepatitis, thereby promoting the rapid decomposition of ethanol and reducing ethanolinduced liver damage. By up-regulating the expression of Nrf2 protein in liver tissue with alcoholic liver injury and increasing amounts of nuclear transfer, the expression of downstream antioxidant proteins are further activated and the oxidation defense system (Zhang et al. 2000; Hong et al. 2016) is activated so as to achieve liver protection.

By up-regulating the expression of antioxidant protein HO-1 in the liver of rats with alcoholic hepatitis, antioxidative and anti-apoptotic capabilities are enhanced, cell survival in liver tissues is improved, and the oxidation defense system is enhanced. SRA inhibited the degradation of IKBA protein in liver tissue of rats with alcoholic liver injury and reduced the release of NF-KB p65 (Chen et al. 2011; Huang et al. 2017), thereby inhibiting inflammation. SRA inhibited levels of NF-kB p 65 in alcoholic liver injury tissue and reduced the release of inflammatory factors, thereby inhibiting inflammation.

\section{Conclusion}

Succinyl rotundic acid reduces inflammatory reactions by reducing the levels of NOS and NO, regulating levels of Bcl-2, Bax, and Caspase- 3 , and regulating NF-kB and antioxidative stress pathways. It has an antagonistic effect on alcoholic liver injury and has potential for treating clinical alcoholic liver disease. 


\section{Acknowledgements}

This study was supported by the National Natural Science Foundation of China (grant no. 31470418). Jilin Province Administration of Traditional Chinese Medicine Project (grant no. 2020051).

\section{Author Contributions}

Yajie Zhang: Overall instructor; Yufang He, Fang Xia, Xu Wang: Responsible for the experiment and operation; Lijing $\mathrm{Li}$ and Minlun Nan: Experimental operation support.

\section{References}

Baghdasaryan N, G Ayvazyan, M Grigoryan, L Avetisyan, O Asatryan, N Mnatsakanyan A Perikhanyan (2019). Liver involvement in the process of acute respiratory infections in pediatric patients. $J$ Infect Dev Countr 13:63S-68S

Chen KH, BR Lin, CT Chien (2011). Emblica officinalis Gaertn.attentuates nitrosodiethylamine-induced apoptosis, autophagy, and inflammation in rat livers. J Med Food 14:746-755

Ciardullo S, E Muraca, S Perra, E Bianconi, F Zerbini, A Oltolini, R Cannistraci, P Parmeggiani, G Manzoni, A Gastaldelli, G Lattuada, G Perseghin (2020). Screening for non-alcoholic fatty liver disease in type 2 diabetes using non-invasive scores and association with diabetic complications. BMJ Open Diab Res Care 8; Article e000904

He Z, J Chen, J Wang, L Xu, Z Zhou, M Chen, Y Zhang, M Shi (2020). Expression of hepatitis B surface antigen in liver tissues can serve as a predictor of prognosis for hepatitis B virus-related hepatocellular carcinoma patients after liver resection. Eur J Gastroenterol Hepatol 33:76-82

Hong HM, MT Chen, YY Wang (2016). Research progress of liver apoptosis induced by decreasing nitric oxide. J Pract Med 32:1533-1535

Hsu YM, YC Hung, LH Hu, YJ Lee, MC Yin (2015). Anti-diabetic effects of madecassic acid and rotundic acid. Nutrients 7:10065-10075

Huang QH, LQ Xu, YH Liu, JZ Wu, X Wu, XP Lai, YC Li, ZR Su, JN Chen, YL Xie (2017). Polydatin protects rat liver against ethanolinduced injury: Involvement of CYP2E1/ROS/Nrf2 and TLR4/NFkB p65 pathway. Evid Based Complem Altern Med 2017; Article 7953850
Jiao W, X Zhao, G Wu, X Zhang, H Wu, Y Cui (2020). Bioactivation of lumiracoxib in human liver microsomes: Formation of GSH- and amino adducts through acyl glucuronide. Drug Test Anal 12:827-835

Klemm K, C Eipel, D Cantre, K Abshagen, MD Menger, B Vollmar (2008). Multiple doses of erythropoietin impair liver regeneration by increasing TNF - alpha, the Bax to Bcl-xL ratio and apoptotic cell death. PLoS One 3; Article e3924

Li JJ, FF Chen, LY Song, JH Zhu, RM Yu (2017). Study progress on antitumor activities of artemisinin and its derivatives. Chin J Biochem Pharm 37:10-14

Li YM, JG Fan, BY Wang (2011). Guidelines for management of alcoholic liver disease: An updated and revised edition. Mod Med Health 27:801-804

Mao DW, YQ Chen, L Wang (2008). Relationship of Caspase-8 and Caspase-3 to apoptosis. J Liaon Univ Trad Chin Med 10:148-150

Maryconi MJ, SC Mitchell (2014). Therapy for alcoholic liver disease. World J Gastroenterol 20:2143-2158

Noto D, F DiGaudio, IG Altieri, AB Cefalù, S Indelicato, F Fayer, R Spina, C Scrimali, A Giammanco, A Mattina, S Indelicato, M Greco, D Bongiorno, M Averna (2020). Automated untargeted stable isotope assisted lipidomics of liver cells on high glucose shows alteration of sphingolipid kinetics. Biochim Biophys Acta Mol Cell Biol Lipids 1865; Article 158656

Raisova M, AM Hossini, J Eberle (2001). The Bax/Bcl - 2 ratio determines the susceptibility of human melanoma cells to CD95/ Fas-mediated apoptosis. J Invest Dermatol 117:333-340

Sehgal R, H Singh, IP Singh (2020). Comparative study of spironolactone and eplerenone in management of I ascites in patients of cirrhosis of liver. Eur J Gastroenterol Hepatol 32:535-539

Tang Y, CB Forsyth, A Farhadi (2009). Nitric oxide mediated intestinal injury is required for alcohol induced gut leakiness and liver damage. Alcohol Clin Exp Res 33:1220-1230

Wei L, XL Zhang, Z He (2015). Advances in alcoholic liver disease treatment. Chin J Clin 9:2593-2597

Wu LH, MH Chen, JY Cai, Y Yuan, LQ Wu, HM Zhou, L Li, K Wan, XX $\mathrm{He}$ (2020). The correlation between intestinal mucosal lesions and hepatic dysfunction in patients without chronic liver disease. Medicine 99; Article e18837

Zhang GW, YY Fan, YJ Bao (2002). Nitric oxide anal liver injury. Prog Anat Sci 28:75-78

Zhang Y, C Goodyer, A Leblanc (2000). Selective and protracted apoptosis in human primary neurons microinjected with active caspase $-3,-6$, - 7 and - 8. J Neurosci 20:8384-8389

Zou H, J Sun, B Wu, Y Yuan, J Gu, J Bian, X Liu, Z Liu (2020). Effects of cadmium and/or lead on autophagy and liver injury in rats. Biol Trace Elem Res 198:206-215 